\title{
Therapie von schwerbehandelbaren chronischen Wunden mit Hyaluronsäureester: Eine Fallbeschreibung über sechs Patient*innen
}

\author{
Emanuel Maitz (D) Barbara Binder (D)
}

Eingegangen: 23. Juli 2020 / Angenommen: 16. Februar 2021 / Online publiziert: 18. März 2021

(C) Der/die Autor(en) 2021

\begin{abstract}
Zusammenfassung Die Behandlung von chronischen Wunden ist meist eine große Herausforderung für alle Beteiligten. Oft ist der Weg bis zur Heilung langwierig und frustran, sodass verschiedene Therapieversuche durchgeführt werden müssen, bis eine Heilung erzielt wird. In dieser retrospektiven Fallbeschreibung berichten wir über den Behandlungserfolg durch die Therapie mit einem Hyaluronsäureester-Flies, den wir bei 6 Patient*innen mit chronischen Wunden unterschiedlicher Genese erzielen konnten. Alle Patient*innen bekamen zusätzlich Kompressionsbandagen oder Kompressionsstrümpfe, zwei zusätzlich eine Druckentlastung und alle wurden, wenn nötig, debridiert. Die chronischen Wunden von 5 der 6 Patient*innen heilten vollständig oder nur mit einem minimalen Restdefekt ab, 1 Patient war weiterhin therapieresistent. Speziell bei Patient*innen mit Grunderkrankungen, welche eine erfolgreiche Therapie besonders erschweren, konnten wir feststellen, dass durch eine Behandlung mit Hyaluronsäureestern dennoch gute Erfolge erzielt werden können.
\end{abstract}

Alle Autoren haben zur Konzeption und Gestaltung der Studie beigetragen. Materialaufbereitung, Datenerfassung und Analyse wurden von Emanuel Maitz und Barbara Binder durchgeführt. Der erste Entwurf des Manuskripts wurde von Emanuel Maitz verfasst und alle Autoren kommentierten frühere Versionen des Manuskripts. Alle Autoren haben das endgültige Manuskript gelesen und genehmigt.

\section{Ass. Dr. E. Maitz}

Univ.-Klinik für Orthopädie und

Traumatologie, Medizinische Universität Graz,

Auenbruggerplatz 5, 8036 Graz, Österreich

PD Dr. B. Binder $(\bowtie)$

Univ.-Klinik für Dermatologie und

Venerologie, Medizinische Universität Graz,

Auenbruggerplatz 8, 8036 Graz, Österreich

barbara.binder@klinikum-graz.at
Schlüsselwörter Hyaluronsäureester . Hyaluronsäureester-Flies · Chronische Wunden · Hyalofill ${ }^{\circledR} \cdot$ Hyaff ${ }^{\circledR}$

Treatment of chronic hard-to-heal wounds with hyaloronic acid ester: a case series of six patients

Summary The treatment of chronic wounds is usually a major challenge for the involved medical staff. Various different treatment options have to be tested achieving a satisfactory result. In this retrospective case report, we describe successful treatments with hyaluronan acid ester fleece in six patients with chronic wounds of different origins. Furthermore, all patients received compression bandages or compression stockings, two patients were treated additionally with pressure relief devices and all were debrided if necessary. The chronic wounds of five out of the six patients healed completely over a period of $1.5-11$ months or only with a minimal residual defect. However, one patient was still resistant to therapy over five months. In summary, the treatment with hyaluronan esters achieved good results especially in patients with complex medical history that makes a successful therapy particularly difficult.

Keywords Hyaloronic acid ester - Hyaloronic acid ester fleeze · Chronic wounds · Hyalofill ${ }^{\circledR}$. Hyaff ${ }^{\circledR}$

\section{Einleitung}

Über eine allgemein gültige Definition von chronischen Wunden wird noch diskutiert [1]. Jedoch hat die Deutsche Gesellschaft für Wundheilung und Wundbehandlung in der S3-Leitlinie mit Stand 2012 eine Wunde als chronisch definiert, wenn die Wunde mit einem Integritätsverlust der Haut und einer oder mehrerer 
Tab. 1 Beschreibung der Wunden anhand von Lokalisation, Art, Größe, Bestehungs-, und Behandlungszeit

\begin{tabular}{|c|c|c|c|c|c|c|}
\hline Patient & Lokalisation & Art & $\begin{array}{l}\text { Seit wann } \\
\text { bestehend }\end{array}$ & $\begin{array}{l}\text { Größe am Beginn der } \\
\text { Behandlung }\end{array}$ & $\begin{array}{l}\text { Behandlungszeit mit Hyalu- } \\
\text { ronsäureester }\end{array}$ & Abgeheilt \\
\hline Patient 1 & Innenknöchel rechts & Ulcus cruris venosum & 27 Monate & $3,8 \times 0,8 \mathrm{~cm}$ & 11 Monate & $\mathrm{Ja}$ \\
\hline Patient 2 & $\begin{array}{l}\text { Großzehenballen } \\
\text { links }\end{array}$ & $\begin{array}{l}\text { Diabetisches Fußsyndrom und } \\
\text { venöse Insuffizienz }\end{array}$ & 6 Monate & $1,5 \mathrm{~cm}$ lang & 7 Monate & $\mathrm{Ja}$ \\
\hline Patientin 3 & $\begin{array}{l}\text { Äußerer Unterschen- } \\
\text { kel rechts }\end{array}$ & Ulcus cruris venosum & 34 Monate & $2 \times 2 \mathrm{~cm}$ & 4 Monate & $\begin{array}{l}\text { Ja, nach weiteren } 2 \text { Mo- } \\
\text { naten }\end{array}$ \\
\hline Patientin 4 & Unterschenkel rechts & $\begin{array}{l}\text { Traumatisches Ulcus, Varico- } \\
\text { sis }\end{array}$ & 4 Monate & $3,5 \times 4 \mathrm{~cm}$ & 1,5 Monate & $\mathrm{Ja}$ \\
\hline Patient 5 & $\begin{array}{l}\text { Großzehenballen } \\
\text { rechts }\end{array}$ & Malum perforans & 14 Monate & $3 \times 2 \mathrm{~cm}$ & 5 Monate & $\begin{array}{l}\text { Restdefekt ( } 3 \mathrm{~mm} \text { lang, } \\
3 \mathrm{~mm} \text { tief) }\end{array}$ \\
\hline Patient 6 & Innenknöchel links & Ulcus cruris venosum & $>$ 10 Jahre & $3 \times 5,5 \mathrm{~cm}$ & 5 Monate & Nein, keine Besserung \\
\hline
\end{tabular}

darunter liegenden Strukturen einhergeht und nicht innerhalb von acht Wochen abheilt [2]. Aufgrund der unterschiedlichen Definitionen ist es schwierig, eine exakte Prävalenz anzugeben. [2-4].

2016 wurde von Heyer et al. eine Analyse der chronischen Wunden in Deutschland veröffentlicht, die zeigen konnte, dass chronische Ulzerationen am Unterschenkel (venöse, arterielle, gemischte und nicht spezifierte Ulcera) mit einer Prävalenz von $0,70 \%$ die häufigste, und diabetische Fußulcera mit $0,27 \%$ die zweithäufigste Ursache sind; daneben Druckulcera mit $0,18 \%$ und Ulcera anderer Entitäten wie Pyoderma gangrenosum und Gangrän mit 0,03\% [3]. Die Lebensqualität wird durch diese chronischen Wunden, inklusive sozialer Ausgrenzung, negativ beeinflusst $[2,5-8]$. Hinzu kommt der große therapeutische und pflegerische Aufwand, der mit hohen Kosten für das Gesundheitssystem und die Gesellschaft allgemein einhergeht [9-11]. Tritt eine Stagnation während der Behandlung auf, wird die Situation noch zusätzlich verschlechtert. Die Wahl des entsprechenden lokaltherapeutischen Produktes kann eine therapeutische Herausforderung darstellen [1, 12]. Hyaluronsäurehaltige Wundauflagen, in verschiedenen Formen angeboten, können in solchen Fällen die Wundheilung positiv beeinflussen [13].

Hyaluronsäure oder auch Hyaluronan (HA) ist der Hauptbestandteil der extrazellulären Matrix. Es ist ein Glykosaminoglykan, also ein langkettiges, lineares Polysaccharid mit einem Molekulargewicht von 105 bis 107 Dalton. Es ist unter anderem Hauptbestandteil des Gallertkerns der Bandscheiben, der Synovia und des Glaskörpers [1, 13, 14]. Als Teil der extrazellulären Matrix hat es einen entscheidenden Anteil an allen Prozessen in der Wundheilung. Es stimuliert die epitheliale Zellproliferation und -migration, weiters steigert es die Bildung von Granulationsgewebe und reduziert die Entzündungsreaktion [15, 16].

Mit den folgenden Fallbeschreibungen wollen wir die Wirksamkeit der Behandlung mit einem Hyaluronsäureester-Flies bei therapieresistenten chronischen Wunden darstellen.

\section{Material, Methoden, Ergebnisse}

Es wurden 6 Patient*innen im Alter zwischen 38 und 83 Jahren retrospektiv beschrieben, die im Zeitraum von April 2015 bis August 2017 in unserer dermatologischen Gefäßambulanz mit Hyaluronsäureester-Flies behandelt wurden (EK-Nummer: 30-277 ex 17/18). Als Datenquellen dienten die schriftlichen Ambulanzbefunde, Fotodokumentationen und Planimetrien.

Angewandt wurde Hyalofill ${ }^{\circledR}$-F (Hyaff ${ }^{\circledR} /$ absorbierende Hautmatrix; der Firma Anika Therapeutics, Padua, Italien), ein $100 \%$ Ester der Hyaluronsäure entsprechend den Anwendungshinweisen des Erzeugers. Als Sekundärverband wurde ein Polyurethanschaumstoff appliziert.

Eingeschlossen wurden 2 Frauen und 4 Männer im Alter zwischen 38 und 83 Jahren mit chronischen Ulcera an den Unterschenkeln oder Füßen, welche seit mindestens 4 Monaten bestanden. Die $\mathrm{zu}$ Grunde liegenden Ursachen der Ulcera waren dabei unterschiedlich: 3 venöse Ulcera, 1 diabetisches Fußsyndrom, 1 Ulcus bei peripherer Polyneuropathie und 1 posttraumatisches Ulcus (Tab. 1). Bei jedem Patienten kam das Hyalofill ${ }^{\circledR}-\mathrm{F}$ in der Granulations- und Epithelisationsphase zum Einsatz. Vor Behandlungsbeginn wurde bei allen Patient*innen eine ausführliche Anamnese inkl. Begleiterkrankungen erhoben, ebenso die aktuelle Medikation und Allergieanamnese. Laboruntersuchungen fanden nicht statt. Ein arterieller Doppler wurde durchgeführt und der arterielle Dopplerindex (ABI) berechnet. Bei allen beschriebenen Patient*innen war der ABI $>1$, sodass eine klinisch relevante arterielle Durchblutungsstörung ausgeschlossen werden konnte. Regelmäßige Verbandswechsel (2-3×/Woche) durch Hauskrankenpflege, Patient*innen oder deren Angehörige bzw. beim Allgemeinmediziner und ärztliche Kontrollen in unserer Ambulanz (Patientin 4 anfangs $1 \times /$ Woche, ansonsten alle 2-4 Wochen) wurden vor, während und nach dem Beobachtungszeitraum durchgeführt. Alle Patient*innen bekamen Kompressionsbandagen oder Kompressionsstrümpfe und wurden, wenn nötig, chirurgisch debridiert (mit dem scharfen Löffel oder einer Ringkürette nach vorheriger Oberflächenanästhesie mit einem Lidocain-Spray). Zusätzlich erfolgte 


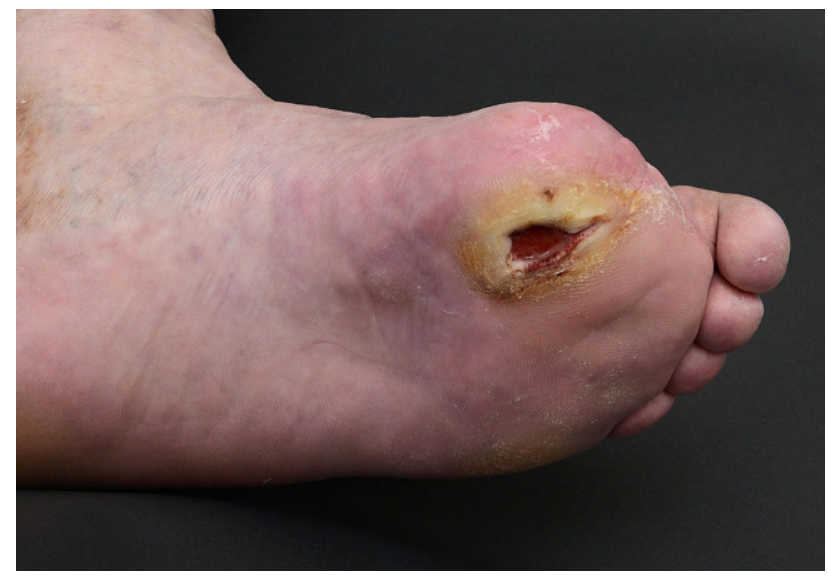

Abb. 1 Patient 2 vor Therapie

eine Druckentlastung bei Patient 2 und 5 mittels orthopädischem Verbandsschuh; ansonsten wurde keine zusätzliche Begleittherapie parallel durchgeführt.

\section{Patient*innendetails}

Pat 1: 77a, männlich, Raucher seit 40 Jahren, venöses Ulcus cruris am rechten Innenknöchel bestehend über 27 Monate. Der Patient war bereits mit verschiedenen Lokaltherapien behandelt worden; zuletzt durchgeführte Lokaltherapie: Hydrofaserverband. Nach elf Monaten Behandlung mit Hyaluronsäureester heilte das Ulcus vollständig ab.

Pat 2: 60a, männlich, 2005 wurde die linke Großzehe wegen einer Osteomyelitis amputiert. Bevor er mit Hyaluronsäureester behandelt wurde, bestand seit sechs Monaten ein tiefes, spaltförmiges Ulcus an der Amputationsstelle. Der Patient hatte sich selbst therapiert. Zuletzt durchgeführte Lokaltherapie: Silbersulfdiazin-Gel und Schaumstoff. Sieben Monate nach Beginn der Hyaluronsäureesterbehandlung heilte dieses ab, (Abb. 1 und 2).

Pat 3: 83a, weiblich, seit 34 Monaten bestehendes Ulcus cruris venosum am rechten Außenknöchel.

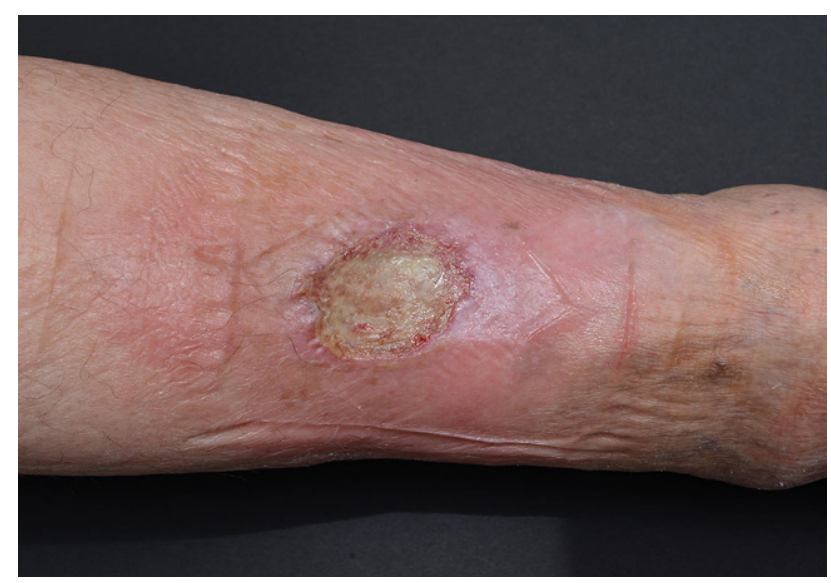

Abb. 3 Patient 4 vor Therapie

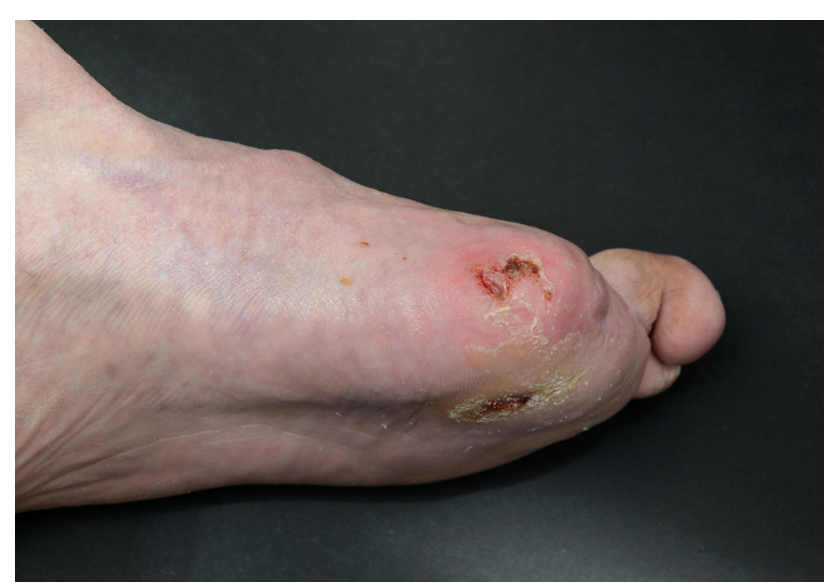

Abb. 2 Patient 2 nach Therapie

Zuletzt durchgeführte Lokaltherapie: Medizinischer Honig, Hydrofaserverband und Superabsorber. Nach vier Monaten Therapie mit Hyaluronsäureester, wurde auf Wunsch der Patientin wegen zeitweiser Schmerzen der Hyaluronsäureester abgesetzt und es erfolgte anschließend drei Monate eine Therapie mit dem bisher verwendeten Polyurethanschaumstoffverband mit vollständiger Abheilung.

Pat 4: 76a, weiblich, mit einem traumatisch bedingten Ulcus cruris rechts, durch eine unbehandelte Varicosis begünstigt, welcher sich etwa vier Monate in einem therapieresistenten Zustand befand. Die bisherige Therapie wurde extern durchgeführt ohne entsprechende Kompressionstherapie. Zuletzt durchgeführte Lokaltherapie: Hydrofaserverband+Silber und Schaumstoff+Silber. Eine Abheilung konnte nach eineinhalb Monaten Therapie mit Hyaluronsäureester erzielt werden (Abb. 3 und 4).

Pat 5: 38a, männlich, mit Malum perforans am rechten Großzehenballen (seit über einem Jahr), mit Sondierung bis zum Knochen (eine Osteomyelitis wurde radiologisch ausgeschlossen), bedingt durch ein diabetisches Fußsyndrom. Zuletzt durchgeführte Lokaltherapie: Hydrofaserverband+Silber. Es wurde fünf Monate mit Hyaluronsäureester behandelt, bis

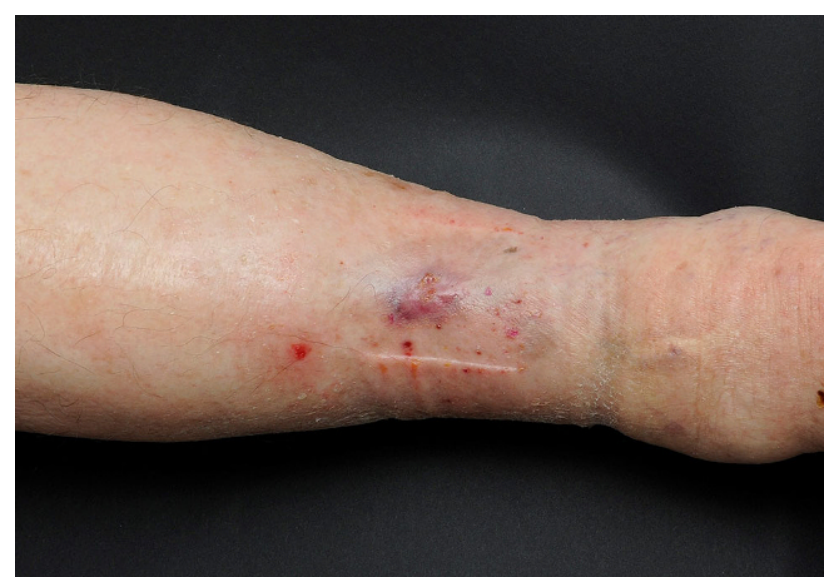

Abb. 4 Patient 4 nach Therapie 
eine weitgehende Abheilung mit einem $3 \mathrm{~mm}$ langem und $3 \mathrm{~mm}$ tiefem Restdefekt, erzielt werden konnte.

Pat 6: 64a, männlich, mit Ulcus cruris venosum, bestehend seit mehr als 10 Jahren, konnte auch nach fünf Monaten Therapie mit Hyaluronsäureester keine deutliche Befundbesserung erreicht werden. Zuletzt durchgeführte Lokaltherapie: polyabsorbierendes Polyacrylat. Die Wundheilung wurde durch eine Adipositas permagna und latente cardiale Dekompensation, wobei die Kompressionstherapie ausgesetzt werden musste, negativ beeinflusst.

\section{Diskussion}

Speziell behandlungsresistente chronische Ulcera sind oft, sowohl für den Patienten/die Patientin und dessen/deren Angehörige, als auch für die behandelnden Mediziner*innen und die Pflege, ein großes und nicht selten herausforderndes Problem. In vielen Fällen ist nicht ersichtlich, warum keine Abheilung trotz phasengerechter Therapie erzielt werden kann. Mit Hyaluronsäureester konnten wir eine Verbesserung bis hin zur Abheilung erzielen. 4 von 6 heilten vollständig ab, Patient 5 zeigte nach 5 Monaten einen schlitzförmigen, $3 \mathrm{~mm}$ tiefen Restdefekt und kam nicht mehr zum weiteren Follow-up. Bei Patient 6 zeigte sich ein frustraner Therapieversuch aufgrund seiner Begleiterkrankungen und daher inkonsequenten Kompressionstherapie. Durch das angewandte Hyaluronsäureester-Flies konnten bei oben beschriebenen Patient*innen die Wunden aus der Stagnation befreit werden und der Heilungsprozess einsetzen. Limitierend in dieser Fallübersicht ist sicher die geringe Patient*innenzahl und die retrospektive Analyse der Daten. Außerdem ist das Resultat bei der Behandlung chronischer Wunden immer stark von der individuellen Compliance der Patient*innen abhängig und damit nur bedingt vergleichbar.

Einige Studien und Fallbeschreibungen konnten die Wirksamkeit von Hyaluronsäureestern bereits bestätigen [13-22]. Speziell bei Patient*innen mit Grunderkrankungen, die eine erfolgreiche Therapie besonders erschweren, zeigen diese, dass durch eine Behandlung mit Hyaluronsäureestern dennoch gute Erfolge erzielt werden können [13-18]. Eine Metaanalyse von Voigt und Driver untersuchte den Behandlungserfolg mit Hyaluronsäure bei Verbrennungen, chirurgischen epithelialen Wunden und chronischen Wunden, wobei sie aufzeigen konnten, dass bei 8 von 9 Studien ein signifikanter Erfolg erzielt werden konnte [23].

\section{Zusammenfassung}

Mit den dargestellten Fallbeschreibungen konnte gezeigt werden, dass mit diesem HyaluronsäureesterFlies eine Abheilung bzw. Verbesserung der Wundheilung bei therapieresistenten chronischen Wunden erzielt werden kann. Dennoch muss berücksichtigt werden, dass eine gewisse Behandlungszeit und Patient*innenmitarbeit nötig ist, um einen gewünschten Therapieerfolg zu erzielen. Hyaluronsäureester stellen jedoch eine zielführende Therapieoption für chronische Wunden dar, welche in der Heilung stagnieren und keine oder nur geringe Besserung unter der bisherigen Lokaltherapie zeigen. Randomisiert-kontrollierte, prospektive klinische Studien sind notwendig, um die Vorteile gegenüber bisher verwendeten Lokaltherapeutika empirisch zu belegen.

Funding Open access funding provided by Medical University of Graz.

Interessenkonflikt B. Binder und E. Maitz geben an, dass kein Interessenkonflikt besteht.

Open Access Dieser Artikel wird unter der Creative Commons Namensnennung 4.0 International Lizenz veröffentlicht, welche die Nutzung, Vervielfältigung, Bearbeitung, Verbreitung und Wiedergabe in jeglichem Medium und Format erlaubt, sofern Sie den/die ursprünglichen Autor(en) und die Quelle ordnungsgemäß nennen, einen Link zur Creative Commons Lizenz beifügen und angeben, ob Änderungen vorgenommen wurden.

Die in diesem Artikel enthaltenen Bilder und sonstiges Drittmaterial unterliegen ebenfalls der genannten Creative Commons Lizenz, sofern sich aus der Abbildungslegende nichts anderes ergibt. Sofern das betreffende Material nicht unter der genannten Creative Commons Lizenz steht und die betreffende Handlung nicht nach gesetzlichen Vorschriften erlaubt ist, ist für die oben aufgeführten Weiterverwendungen des Materials die Einwilligung des jeweiligen Rechteinhabers einzuholen.

Weitere Details zur Lizenz entnehmen Sie bitte der Lizenzinformation auf http://creativecommons.org/licenses/by/4. $0 /$ deed.de.

\section{Literatur}

1. Dissemond J, Augustin M, Eming SA, et al. Moderne Wundtherapie - Praktische Aspekte der lokalen, nicht- interventionellen Behandlung von Patienten mit chronischen Wunden.Jddg-J Ger Soc Dermatology. 2014;12(7):541-55.

2. S3-Leitlinie:A.Lokaltherapie chronischerWunden bei Patienten mitden Risiken periphere arterielleVerschlusskrankheit, Diabetes mellitus, chronische venöse Insuffizienz. DGfW. 2012;09(1):1-279.

3. Heyer K, Herberger K, ProtzK, et al.Epidemiology of chronic wounds in Germany: Analysis of statutory health insurance data. Wound Repair Regen.2016;24(2):434-42.

4. Firth J, Nelson EA, Hale C, et al. A review of design and reporting issues in self-reported prevalence studies of leg ulceration. J Clin Epidemiol. 2010;1;63(8):907-13. Aug.

5. Charles H. Does leg ulcer treatment improve patients' quality oflife? JWound Care. 2004;1;13(6):209-13. Jun.

6 . Suzanne K, Charne M, Kylie E. The impact of providing product funding for compression bandaging and medical footwear on compression use, wound healing and quality of life. Int Wound J.2011;16;9(5):494-504.Dec.

7. Green J, Jester R. Health-related quality of life and chronic venous leg ulceration: part 2. Br J Community Nurs. 2010;1;15(Sup1):S4-14. Mar.

8. Augustin M, Brocatti L, Rustenbach S, et al. Quality of life evaluation in wounds: validation of theFreiburgLifeQuality 


\section{fallbericht}

Assessment-wound module, a disease-specificinstrument. Int Wound J. 2010;30;7(6):493-501. Sep.

9. Augustin M, Brocatti L, Rustenbach S, et al. Cost-ofillness of leg ulcers in the community. Int Wound J. 2012;1;11(3):283-92. Oct.

10. Rabe E, Pannier F. Societal costs of chronic venous disease in CEAP C4, C5, C6 disease. Phlebology. 2010;24;25(1_suppl):64-7.Sep.

11. Purwins S, Herberger K, Debus E, et al. Cost-of-illness of chronic leg ulcers in Germany. Int Wound J. 2010;19;7(2):97-102.Apr.

12. Murray RZ, West ZE, Cowin AJ, et al. Development and use of biomaterials as wound healing therapies. Burns Trauma. 2019;25(7):2. https://doi.org/10.1186/s41038-018-0139-7.

13. Ballard K, Cantor AJ. Treating recalcitrant diabetic wounds with hyaluronic acid: a review of patients. Ostomy Wound Manage. 2003;49(4):37-8.40,42, passim.

14. Ghazi K, Deng-Pichon U. Warnet et al. Hyaluronan Fragments Improve Wound Healing on In Vitro Cutaneous Model through P2X7 Purinoreceptor Basal Activation: Role of Molecular Weight. Plos One. 2012;7:11.

15. Chen WYJ, Abatangelo G. Functions of hyaluronan in wound repair. Wound Repair Regen. 1999;7(2):79-89.

16. Muto J, Sayama K, Gallo RL, et al. Emerging evidence for essential role of hyalurnan in cutaneous biology. J Dermatol Sci. 2019;94:190-5.
17. Vazquez JR, Short B, Findlow AH, et al. Outcomes of hyaluronan therapy in diabetic foot wounds. Diabetes Res Clin Pract. 2018;16;59(2):123-7. Feb.

18. Nyman E, Henrikson J, Ghafouri B, et al. Hyluronic acid accelerates re-epithelialisation and alters protein expression in a human wound model. Plast Reconstr Surg Glob Open. 2019;7:e2221.

19. Gupta RC, Lall R, Srivastava A, Hyaluronic Acid SA. Molecular Mechanisms and Therapeutic Trajectory. Front Vet Sci. 2019;25;6:192. Jun.

20. Aya KL, Stern R. Hyaluronan in wound healing: rediscovering a major player. Wound Repair Regen. 2014 SepOct;22(5):579-93.

21. Fallacara A, Baldini E, Manfredini S, Vertuani S. Hyaluronic Acid in the Third Millennium. Polymers (Basel). 2018 Jun 25;10(7):701.https://doi.org/10.3390/polym10070701.

22. Neuman MG, Nanau RM, Oruña-Sanchez L, Coto G. Hyaluronic acid and wound healing. J Pharm Pharm Sci. 2015;18(1):53-60.

23. Voigt J, Driver VR. Hyaluronic acid derivatives and their healing effect on burns, epithelial surgical wounds, and chronic wounds: A systematic review and meta-analysis of randomized controlled trials. Wound Repair Regen. 2012;20(3):317-31.

Hinweis des Verlags Der Verlag bleibt in Hinblick auf geografische Zuordnungen und Gebietsbezeichnungen in veröffentlichten Karten und Institutsadressen neutral. 